\title{
NONLINEAR DYNAMICS OF PIPELINE WITH LIQUID IN A VICINITY OF CRITICAL FLOW VELOCITIES
}

\author{
O. Limarchenko, Yu. Vasilevskiy \\ Kiev National University, Ukraine
}

\begin{abstract}
We developed the nonlinear model of pipeline dynamics with high-speed liquid motion. On the basis of the variational methods we constructed the nonlinear discrete model and numerical algorithm for investigation of problems of dynamics and dynamical stability of pipeline. We considered examples of dynamical behavior of the system for different velocities of liquid flowing, including the case of critical velocity of flow, when loss of straight line stability of pipeline is possible.
\end{abstract}

Pipeline with flowing liquid is considered as a system, which consists of elastic pipeline and liquid. We consider that longitudinal motion of liquid is given, liquid is ideal and pipeline is modeled on the basis of beam theory. The objective of the article is development of nonlinear model of dynamics of pipeline with liquid and analysis of its validity on reflection of system behavior for different liquid flow velocities, including the domain of critical velocities, when loss of straight-line shape of pipeline is manifested. This model is aimed at investigation of the system behavior on transient motion. In particular such class of problems is of interest for modeling transient motions of pipelines for accidental break of pipelines, for damping vibrations of pipelines. Construction of mathematical model of the system is done on the basis of the Hamilton-Ostrogradskiy variational principle. It is necessary to note that on the use of this variational principle some methodical difficulties originate due to mixed description of motion of system components, namely, lquid motion is considered in the Euler variables and beam motion is described in the Lagrange variables. At the same time total motion of liquid particles consists of sum of these component motions. This results in the necessity of considering the Lagrange variable of lateral deformation of the beam as $u(x, t)$ (here $x$ is longitudinal coordinate, ends of the beam correspond to coordinates $x=0$ and $x=l, t$ is time) as the Euler variable with further taking into account convective component of acceleration in motion equations.

The Lagrange function for the beam can be given as $[1,2]$

$$
\begin{aligned}
L_{B}= & \frac{1}{2} \mu \int_{o}^{l}\left(\frac{\partial u}{\partial t^{2}}\right)^{2} \sqrt{1+u^{\prime 2}} d x-\frac{1}{2} E J \int_{o}^{l}\left(\frac{\partial^{2} u}{\partial x^{2}}\right)^{2} \sqrt{1+u^{\prime 2}} d x \\
& -E F \int_{0}^{l}\left(\sqrt{1+u^{\prime 2}}-1\right) u^{\prime} u \sqrt{1+u^{\prime 2}} d x,
\end{aligned}
$$


where $l$ beam length, $\mu$ is linear density of beam material, $E J$ is bending rigidity of the beam, $F$ is square of beam cross-section, $z$ is lateral coordinate, $u^{\prime}=\frac{\partial u}{\partial x}$.

We assume that potential energy of liquid is equal to zero. On determination of kinetic energy it is necessary to take into account that particles of liquid participate in complicated motion, which caused by longitudinal flow of liquid and motion of pipeline. In spite of the fact that according to its nature $u$ is the Lagrange variable for pipeline, for liquid the variable $u$ should be considered as the Euler variable, therefore, the expression for velocity and acceleration must include not partial derivative of time, but total one. In this case components of particles velocities for axes $x$ and $z$ can be represented as

$$
V_{x}=V \cos \theta ; \quad V_{z}=V \sin \theta+\frac{d u}{d t} .
$$

Here $V=V(t)$ is a given longitudinal velocity of liquid flow along pipeline, $\theta$ is turn angle of beam element about the $O x$ axis. Here from geometrical considerations and because of $\operatorname{tg} \theta=\frac{\partial u}{\partial x}$ it follows that

$$
\sin \theta=\frac{\frac{\partial u}{\partial x}}{\sqrt{1+u^{\prime 2}}}, \quad \cos \theta=\frac{1}{\sqrt{1+u^{\prime 2}}} .
$$

Now taking into account that $\frac{\partial u}{\partial z}=0$ we obtain $\frac{d u}{d t}=\frac{\partial u}{\partial t}+\frac{\partial u}{\partial x} V_{x}$ and finally

$$
\frac{d u}{d t}=\frac{\partial u}{\partial t}+\frac{V \frac{\partial u}{\partial x}}{\sqrt{1+u^{\prime 2}}}
$$

Finally taking into account that on determination of kinetic energy integration is done over the arc $d s=\sqrt{1+u^{\prime 2}} d x$ the Lagrange function for liquid takes the form (here $\rho$ is linear density of liquid)

$$
L_{F}=\frac{1}{2} \rho \int_{0}^{l}\left\{\left[V^{2}+\left(\frac{d u}{d t}\right)^{2}\right] \sqrt{1+u^{\prime 2}}+2 V \frac{\partial u}{\partial x} \frac{d u}{d t}\right\} d x .
$$

Further we restrict ourselves by the assumption that pipeline deformations are small, so we substitute nonlinear terms in components of the Lagrange function for their polynomial approximations. Here we shall take into account in expressions of the Lagrange function terms up to the fourth order of smallness relative to beam deflection (finally this results in obtaining the motion equations accurate to the third order of smallness). So, we obtain the Lagrange function for the investigated dynamical system as

$$
\begin{aligned}
L= & \frac{1}{2} \mu \int_{0}^{l}\left(\frac{\partial u}{\partial t}\right)^{2} d x+\frac{1}{4} \mu \int_{0}^{l}\left(\frac{\partial u}{\partial t}\right)^{2}\left(\frac{\partial u}{\partial x}\right)^{2} d x-\frac{1}{2} E J \int_{0}^{l}\left(\frac{\partial^{2} u}{\partial x^{2}}\right)^{2} d x \\
& -\frac{1}{4} E J \int_{0}^{l}\left(\frac{\partial^{2} u}{\partial x^{2}}\right)^{2}\left(\frac{\partial u}{\partial x}\right)^{2} d x-\frac{1}{2} E F \int_{0}^{l} u^{\prime 3} u d x+\frac{1}{4} \rho V^{2} \int_{0}^{l}\left(\frac{\partial u}{\partial x}\right)^{2} d x
\end{aligned}
$$




$$
\begin{aligned}
& -\frac{1}{16} \rho V^{2} \int_{0}^{l}\left(\frac{\partial u}{\partial x}\right)^{4} d x+\frac{1}{2} \rho \int_{0}^{l}\left(\frac{d u}{d t}\right)^{2} d x+\frac{1}{4} \rho \int_{0}^{l}\left(\frac{d u}{d t}\right)^{2}\left(\frac{\partial u}{\partial x}\right)^{2} d x+ \\
& +\rho V \int_{0}^{l} \frac{\partial u}{\partial x} \frac{d u}{d t} d x+\frac{1}{2} P F \int_{0}^{l}\left(\frac{\partial u}{\partial x}\right)^{2} d x+\frac{1}{2} \rho V^{2} \int_{0}^{l} d x .
\end{aligned}
$$

Let us analyze nature of terms of the Lagrange function. Two first terms of the Lagrange function represents kinetic energy of the beam. The third and fourth terms, represent, correspondingly, potential energy of bending deformations. Here the second and fourth terms are nonlinear. Potential energy of longitudinal compression (stretching) of beam material is represented by the fifth addend. It is necessary to note that in contrast to results of publication of the book [3] this factor is considered in differential form, but not in a simplified integral one. Five first terms of the Lagrange function correspond to dynamics of pipeline, which is considered on the basis of the beam theory. Let us consider now another component of the Lagrange function, which is connected with liquid. The sixth and seventh (nonlinear) addends are connected with centrifugal forces, caused by liquid motion along arc trajectory. The eighth, ninth and tenth addends of kinetic energy are connected with components caused by non-stationary motion of pipeline. Here a part of this energy is connected namely with velocity of pipeline bending motion and partially with effects caused by centrifugal and Coriolis acceleration (it is necessary to note that analysis shows that Coriolis acceleration does not violate plane motion of the system). The eleventh term of expression for the Lagrange function, which contains the $P F$ multiplier, represents potential energy, which appears owing to internal pressure of liquid. Description of this energy component is done according to results of the publication $[3,4]$. The last term in the relation (1) is actually a constant and it can be eliminated from consideration.

For derivation of the motion equations and dynamic boundary conditions we make use of the Hamilton-Ostrogradskiy variational principle. Let us realize variation of the action function $\delta \int_{t_{1}}^{t_{2}} L d t=0$. We note that derivation of dynamic boundary conditions and internal forces of interaction of pipeline walls with liquid for the considered system is complicated enough problem. However, application of the variational approach makes it possible to obtain these dynamic characteristics automatically as a result of use of traditional technique of variation $[1,2]$.

Let us write the motion equations for the pipeline with liquid, which are derived as a result of variation of the Lagrange function (1)

$$
\begin{aligned}
& \frac{\partial^{2} u}{\partial t^{2}} \tilde{A}(x, t)+\frac{\partial^{4} u}{\partial x^{4}} \tilde{B}(x, t)=\rho V \tilde{C}(x, t)+\rho V^{2} \tilde{D}(x, t)+\rho \dot{V} \tilde{E}(x, t) \\
& +P F \tilde{F}(x, t)+2 \mu \frac{\partial^{2} u}{\partial x \partial t} \frac{\partial u}{\partial x} \frac{\partial u}{\partial t}+\frac{1}{2} \mu\left(\frac{\partial u}{\partial t}\right)^{2} \frac{\partial^{2} u}{\partial x^{2}}+\operatorname{EJ} \tilde{K}(x, t)-E F \tilde{L}(x, t) .
\end{aligned}
$$

Here we used for convenience the following denotations

$$
\tilde{A}=-\mu-\rho-\frac{1}{2}(\mu+\rho)\left(\frac{\partial u}{\partial x}\right)^{2}, \quad \tilde{B}=-E J-\frac{E J}{2}\left(\frac{\partial u}{\partial x}\right)^{2}, \quad \tilde{C}=4 \frac{\partial^{2} u}{\partial x \partial t},
$$




$$
\tilde{D}=-3 \frac{1}{2} \frac{\partial^{2} u}{\partial x^{2}}+8 \frac{1}{4} \frac{\partial^{2} u}{\partial x^{2}}\left(\frac{\partial u}{\partial x}\right)^{2}, \quad \tilde{E}=-2 \frac{\partial u}{\partial x}, \quad \tilde{F}=\frac{\partial^{2} u}{\partial x^{2}}
$$

The motion equations (2) are nonlinear and contain nonlinearities up to the third order of smallness. The generating equation for the equation (2) is the biharmonic equation of beam motion. Analytical solving of this nonlinear problem for different cases of motion excitation is practically impossible, therefore, we state the problem of construction of the approximate method for investigation of dynamic behavior of the system on the basis of the method of modal decomposition $[2,5]$.

Let us consider a problem about construction of discrete model of the system on the basis of the Kantorovich method with use of decomposition of system motion by normal modes (variant of the Kantorovich method)

$$
u(x, t)=\sum_{i} c_{i}(t) A_{i}(x) .
$$

Here $c_{i}(t)$ are amplitude parameters of oscillations, which are functions of time, $A_{i}(x)$ are normal modes, which form complete orthogonal system of functions $[1,6]$.

If we substitute the form of solution (3) into the Lagrange function (2), after integration over spatial variable $x$ we obtain the discrete Lagrange function.

$$
\begin{aligned}
L= & \frac{1}{2} \sum_{i} \dot{c}_{i}^{2} N_{i}+\frac{1}{4} \sum_{i j k l} \dot{c}_{i} \dot{c}_{j} c_{k} c_{l} d_{i j k l}^{2}-\frac{1}{2} \frac{E J}{\rho+\mu} \sum_{i} c_{i}^{2} \kappa_{i}^{4} N_{i}-\frac{1}{4} \frac{E J}{\rho+\mu} \sum_{i j k l} c_{i} c_{j} c_{k} c_{l} d_{i j k l}^{6} \\
& -\frac{1}{2} \frac{E F}{\rho+\mu} \sum_{i j k l} c_{i} c_{j} c_{k} c_{l} d_{i j k l}^{3}-\frac{13}{16} \frac{\rho V^{2}}{\rho+\mu} \sum_{i j k l} c_{i} c_{j} c_{k} c_{l} d_{i j k l}^{4}+\frac{7}{4} \frac{\rho V^{2}}{\rho+\mu} \sum_{i j} c_{i} c_{j} \beta_{i j}^{2} \\
& +\frac{2 \rho V}{\rho+\mu} \sum_{i j} \dot{c}_{i} c_{j} \beta_{i j}^{1}-\frac{1}{2} \frac{P F}{\rho+\mu} \sum_{i j} c_{i} c_{j} \beta_{i j}^{2} .
\end{aligned}
$$

Here we used the following denotations of different coefficients, which represent quadratures of known normal modes and their derivatives on the segment $[0, l]$ and can be easily calculated

$$
\begin{aligned}
& N_{i}=\int_{0}^{l} A_{i}^{2} d x ; \quad d_{i j k l}^{2}=\int_{0}^{l} A_{i}^{\prime} A_{j}^{\prime} A_{k} A_{l} d x ; \quad d_{i j k l}^{6}=\int_{0}^{l} A_{i}^{\prime \prime} A_{j}^{\prime \prime} A_{k}^{\prime} A_{l}^{\prime} d x, \\
& d_{i j k l}^{3}=\int_{0}^{l} A_{i}^{\prime} A_{j}^{\prime} A_{k}^{\prime} A_{l} d x ; \quad d_{i j k l}^{4}=\int_{0}^{l} A_{i}^{\prime} A_{j}^{\prime} A_{k}^{\prime} A_{l}^{\prime} d x ; \quad \beta_{i j}^{1}=\int_{0}^{l} A_{i}^{\prime} A_{j} d x ; \quad \beta_{i j}^{2}=\int_{0}^{l} A_{i}^{\prime} A_{j}^{\prime} d x .
\end{aligned}
$$

Let us construct the Lagrange equations of the second kind for the Lagrange function (4). After solving the obtained system relative to the second derivatives of amplitude parameters, which is done on the basis of methods of nonlinear mechanics with the use of the property that the system of equations relative to amplitude parameters includes only terms of the first and third order of smallness (terms of the second order are absent), we 
obtain the following motion equations for the system

$$
\begin{aligned}
\ddot{c}_{r}= & -\frac{E J}{\rho+\mu} \kappa_{r}^{4} c_{r}+\frac{7}{2} \frac{\rho V^{2}}{(\rho+\mu) N_{r}} \sum_{i} c_{i} \beta_{i r}^{2}+\frac{2 \rho V}{(\rho+\mu) N_{r}} \sum_{i} c_{i}\left(\beta_{r i}^{1}-\beta_{i r}^{1}\right) \\
& -\frac{P F}{(\rho+\mu) N_{r}} \sum_{i} c_{i} \beta_{i r}^{2}-\frac{2 \rho \dot{V}}{(\rho+\mu) N_{r}} \sum_{i} c_{i} \beta_{i r}^{2}-\frac{13}{4} \frac{\rho V^{2}}{(\rho+\mu) N_{r}} \sum_{i j k} c_{i} c_{j} c_{k} d_{i j k r}^{4} \\
& -\sum_{i j k} \dot{c}_{i} \dot{c}_{j} c_{k} \frac{1}{N_{r}}\left(d_{j k i r}^{2}-\frac{1}{2} d_{k r i j}^{2}\right)-\frac{E J}{(\rho+\mu) N_{r}} \sum_{i j k} c_{i} c_{j} c_{k} d_{i j k l}^{6} \\
& -\frac{2 E F}{(\rho+\mu) N_{r}} \sum_{i j k} c_{i} c_{j} c_{k} d_{i j k r}^{3}-\frac{1}{2 N_{r}} \sum_{p j k} c_{j} c_{k}\left(-\frac{E J}{\rho+\mu} \chi_{p}^{4} c_{p}\right. \\
& \left.+\frac{7}{2} \frac{\rho V^{2}}{(\rho+\mu) N_{p}} \sum_{i} c_{i} \beta_{i p}^{2}-\frac{P F}{(\rho+\mu) N_{p}} \sum_{i} c_{i} \beta_{i p}^{2}-\frac{2 \rho \dot{V}}{(\rho+\mu) N_{p}} \sum_{i} c_{i} \beta_{i p}^{2}\right) .
\end{aligned}
$$

The system of equations (6) is equations of nonlinear discrete model of the system pipeline with liquid in amplitude parameters. The first six terms of this system are linear and the rest ones are nonlinear terms of the third order of smallness. These equations by means of introduction of new variables and reduction of order of differentiation can be easily transformed to the Cauchy form. Further this enables the use of numerical methods for investigation of transient modes of motion.

On the basis of the developed approach we investigated behavior of pipeline with liquid for different velocities of liquid flow. Integration of the system of ordinary differential equations was done by the Runge-Kutta method for the model, which includes 10 normal modes.

For examples we accept hinging boundary conditions on edges

$$
\left.u(x, t)\right|_{x=0, l}=0 ;\left.\quad \frac{\partial^{2} u}{\partial x^{2}}\right|_{x=0, l}=0,
$$

however, the developed method can be used for arbitrary cases of pipeline fixing.

We consider hinged from both edges steel pipeline of $1 \mathrm{~m}$ length, internal radius is $2 \mathrm{~cm}$, pipeline thickness is $1 \mathrm{~mm}$. Initial motion of the system is caused by deviation by the second normal mode. Thus, initial conditions of the problem can be represented as

$$
c_{2}(0)=0.02, \quad c_{i}(0)=0, \quad i=\overline{1,10}(i \neq 2) ; \quad \dot{c}_{i}(x, 0)=0, \quad i=\overline{1,10}
$$

In numerical examples we consider motion of liquid with constant velocity, physical parameters for liquid are accepted as for water.

It is known $[5,6]$, that there is critical velocity $V_{c r}^{1}$ of flow in the system pipelineliquid, for which frequency of oscillations of pipeline with flowing liquid turns to be zero. Critical velocity is determined on the basis of linear model and it is threshold of loss of stability of straight-line shape of pipeline. Let us write linearized motion equation for amplitude of the first normal mode

$$
\ddot{c}_{1}=\omega^{2} c_{1},
$$

where on the basis of linearized motion equations the value of frequency is determined as 


$$
\omega^{2}=-\frac{E J}{(\rho+\mu)} \chi_{1}^{4}+\frac{7 \rho V^{2}}{2(\rho+\mu) N_{1}} \beta_{11}^{2}-\frac{P F}{(\rho+\mu) N_{1}} \beta_{11}^{2} .
$$

We obtain critical velocity $V_{c r}^{1}$ from the condition $\omega=0$. Then,

$$
V_{c r}^{1}= \pm \sqrt{\frac{2}{7 \rho}\left(\frac{E J N_{1} \chi_{1}^{4}}{\beta_{11}^{2}}+P F\right)} .
$$

For numerical simulation we consider three cases of liquid motion $1-V=0 ; 2-$ $V=0.5 V_{c r}^{1} ; 3-V=V_{c r}^{1}$.

By analogy with publication [7] we consider initial motion of the system caused by perturbation of only the second normal mode. In the case of $V=0$ redistribution of energy occurs only between the second and sixth modes, which is caused by the property that cubic nonlinearity for frequency of the second mode (it is two times greater than frequency of the first normal mode) promotes interaction with the sixth mode. However, this interaction is weak enough. Oscillation process is stable and during 10 periods of oscillations by the first normal mode criteria of instability were not manifested.

In the case than liquid moves with velocity equal to half of critical velocity, process remains to be stable. Energy is redistributed among all modes, but maximal amplitude parameters are peculiar to the second, initially perturbed mode. Excitations of the first and third mode are substantial (commensurate). The following normal modes are excited insignificantly.

In the case of liquid motion with critical velocity the process changes fundamentally and becomes unstable. Initial deviation by the second normal mode results in oscillations with large amplitudes by the first normal mode (up to $8 \mathrm{~cm}$ on the considered interval of calculations), which increase in time. Thus, for liquid motion in a vicinity of the first critical velocity foreseen by linear model motion instability occurs $[5,6]$. Oscillations by other normal modes are performed with small amplitudes.

In Figures 1-3 we showed variation in time of amplitudes of the first, second and third normal modes. We denote by 1 curves of amplitudes of oscillations for zero velocity of liquid flow, by 2 curves for flow with half of critical velocity and by 3 when liquid flows with critical velocity.

It is necessary to note that for zero velocity of liquid flow the first normal mode is not excited. In the case of half critical velocity oscillations are perturbed insignificantly (about $30 \%$ of the value of amplitude of the second normal mode), however nonlinear non-harmonic law of dependence in time is noticed considerably. Behavior of the system considerably differs in a vicinity of critical velocity. Amplitudes of the first normal mode increase significantly, their contribution becomes dominating, distortion of harmonic law becomes more noticeable (manifestation of superharmonics is evident).

Analysis of variation of amplitude of the second normal mode (Figure 2) shows, that influence of high harmonics is insignificant, dependency of amplitude of this harmonics on liquid velocity is not essential, however, on increase of velocity of liquid flow both decrease of oscillation frequency and intensity of energy redistribution between amplitudes of normal modes are manifested (decreasing character of modulation of curves 2 and 3 testifies this). Curve 3, which corresponds to critical value of velocity indicates considerable modulation of oscillations already on initial stage of process development. 


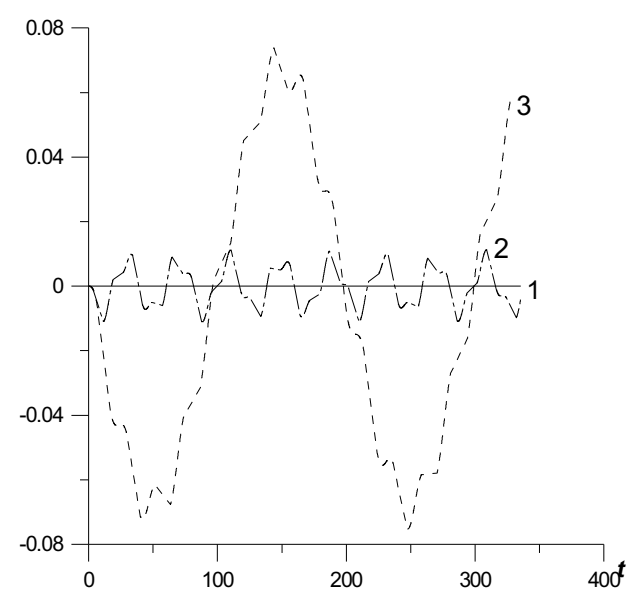

Fig. 1. Amplitudes of the first normal mode

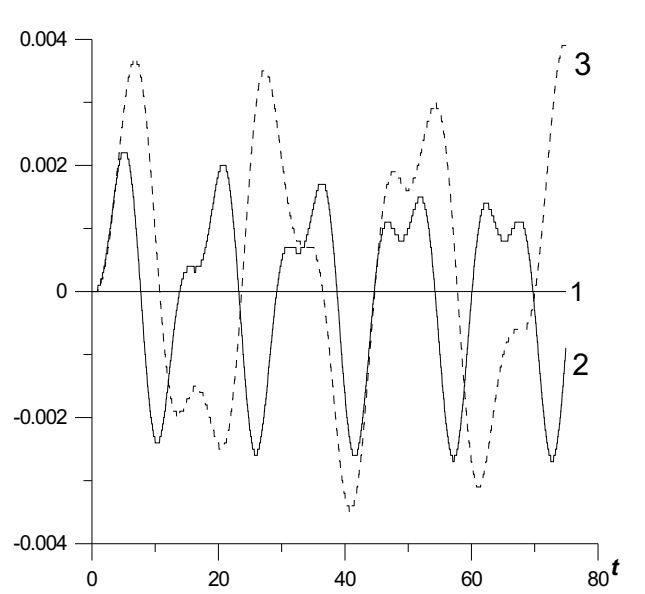

Fig. 3. Amplitudes of the third normal mode

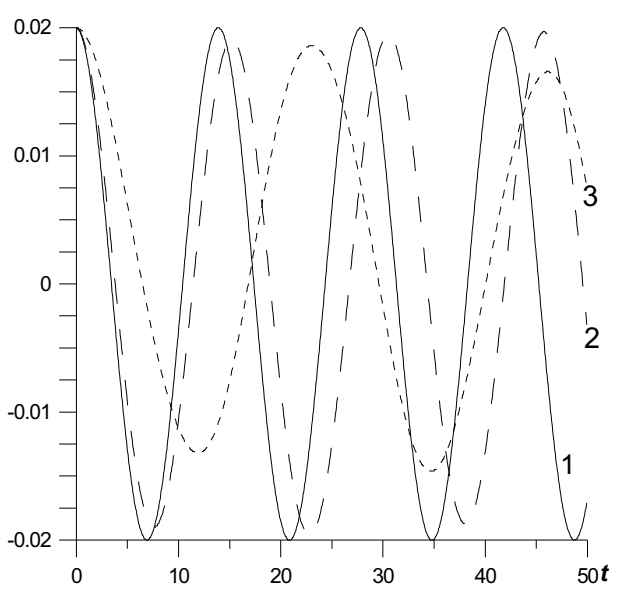

Fig. 2. Amplitudes of the second normal mode

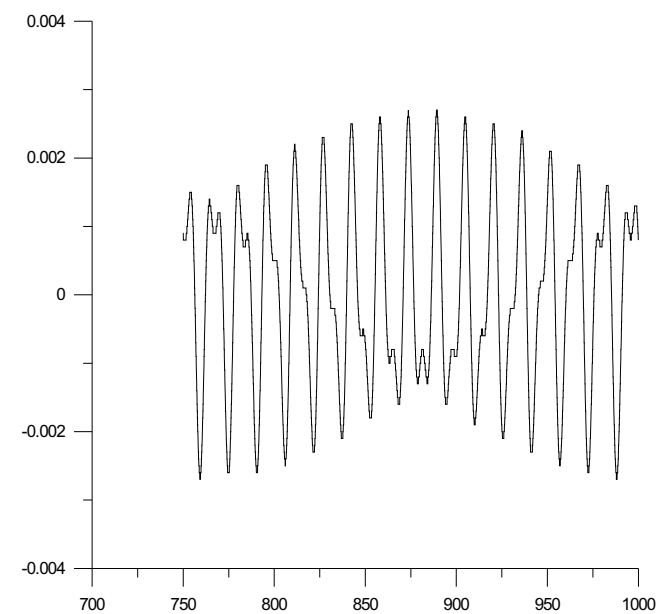

Fig. 4. Influence of superharmonics on amplitudes of the third normal mode

On analysis of variation of the third normal mode amplitude it is seen that manifestation of superharmonics is considerable. If we consider oscillations for greater time interval ( 15 periods of oscillations by the first normal mode), then it is clearly seen (Figure 4) that influence of harmonics with higher frequencies is evident. This effect is caused by manifestation of internal combination resonance, which further promotes the observed energy redistribution.

In Figure 5 for increased time interval we showed effect of modulation of oscillations of amplitude of the third normal mode in time. Modulation effects for other normal modes are manifested weaker. The presence of modulation confirms the necessity of simulation of system behavior on the basis of multimodal approach. This approach is especially significant on studying transient modes of the system motion, when, first, the external disturbance has multi-frequency character, and, second, intense variation of dynamical parameters of the system requires including into consideration high-frequency normal modes, 
which must provide high steepness of variation of parameters of oscillations in the system.

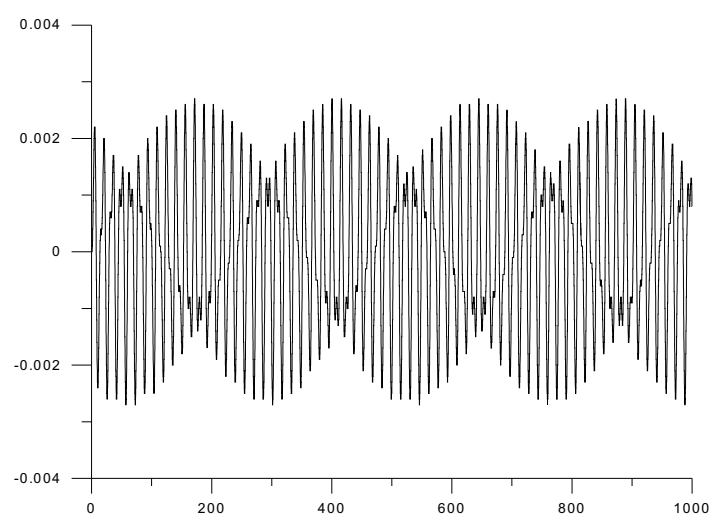

Fig. 5. Manifestation of the effect of modulation for the third normal mode

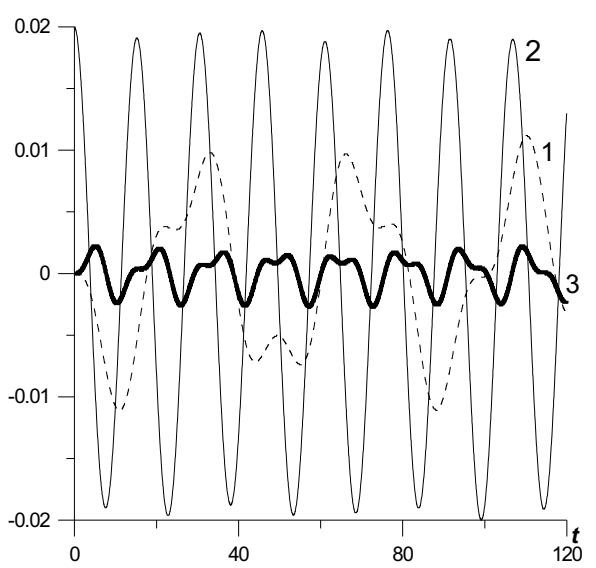

Fig. 6. Amplitudes $c_{1}, c_{2}, c_{3}$ for $V=0.5 V_{\text {cr }}^{1}$

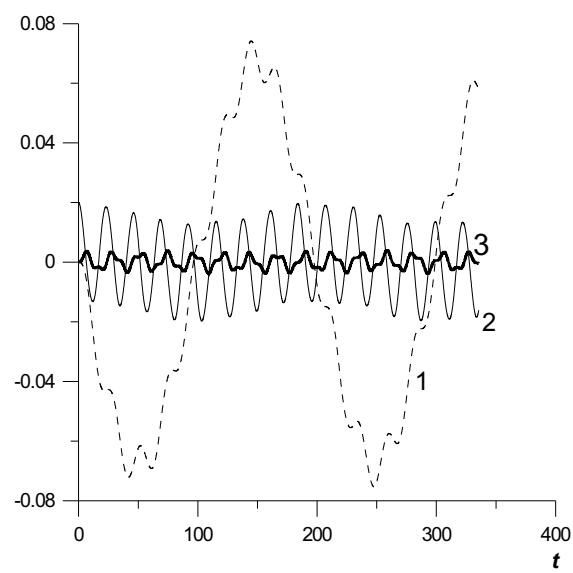

Fig. 7. Amplitudes $c_{1}, c_{2}, c_{3}$ for $V=V_{\text {cr }}^{1}$

For all three cases oscillations caused by initial excitation of the second normal mode are prevalent. However, in the case of system motion in a vicinity of critical velocity the first normal mode (symmetric) dominates, namely growth of which results in loss of stability of straight-line shape of pipeline with liquid.

Variations in time of amplitudes of three first normal modes of oscillations (numbers of curves correspond to the numbers of normal modes) for half critical and critical velocities of liquid flow are shown in Figures 6-7 correspondingly. Then velocity of flow does not tend to critical one oscillations by the second normal modes dominate, energy redistribution in the system promotes nonlinear mechanism of excitation of the first and third normal modes, which is reflected in character of their dependence in time. Influence of superharmonics manifests considerably in variation of the first and third normal modes, 
for the second normal mode such influence is of less significance, and its variation in time differs from harmonic law slightly.

On approach to critical velocities of liquid flow character of system behavior changes considerably. Amplitude of the first normal mode becomes major, while the second and third normal mode are smaller and their average become changeable and values vary in concordance with changes of the first normal mode. Contribution of the third normal mode is insignificant, however it shows complicated nonlinear mechanisms of excitation of oscillations.

For all examples manifestation of normal modes amplitudes interdependency and energy redistribution is considerable. This property reflects development of nonlinear mechanisms of interaction in the system, which essentially intensify due to liquid motion. At the same time processes become more complicated in a vicinity of critical velocities of liquid flow because of manifestation of instability of correspondent normal modes. Although this instability is predetermined by linear effects, increase of amplitudes of oscillations result in manifestation of nonlinear mechanisms of interaction, therefore, to new mechanisms of energy redistribution.

\section{CONCLUSIONS}

On the basis of variational statement of the problem about motion of elastic pipeline with flowing liquid, methods of modal and amplitude decomposition of motions we constructed nonlinear discrete model of system dynamics (nonlinear system of ordinary differential equations relative to amplitude parameters). The model is aimed at investigation of transient modes of system motion and includes great number of normal modes of system oscillations (for numerical examples we consider ten first normal modes). The considered problems testify expediency of taking into account the great number of normal modes. In particular, namely nonlinear interaction between normal modes defines effects of modulation of oscillations and the present of superharmonics in resulting laws of variation of amplitudes. For initial excitation of oscillations by the first anti-symmetric mode (the second mode of oscillations) oscillations by the sixth normal mode are excited significantly even in the case of the absence of liquid flow, which is caused by frequency relation between these normal modes and the presence of cubic nonlinearities. In the case of liquid flow nonlinear mechanisms become stronger and all normal modes entrained in motion, but the first and third normal mode are excited more significantly.

The developed nonlinear mathematical model of pipeline with liquid leads to results, which are in qualitative concordance with real phenomena. Analysis of energy redistribution between normal modes of pipeline oscillations indicates significant influence of liquid flow velocity on character of manifestation of nonlinear mechanisms. Behavior of the system becomes more complicated when velocity of flow tends to critical value. In this case liquid flow promotes increase of pipeline deformation mainly be the first normal mode, which becomes dominating, and mutual influence of normal modes and energy redistribution become stronger.

We plan to use the developed mathematical model for analysis of dynamical phenomena on pipeline break, as well as for working-off new promising types of damping devices (unilateral friction forủes and unilateral recovering forces for dynamic off-tuning of resonant phenomena $[7,8]$ ) with the purpose of reduction of negative influence of tending velocity to critical value, as well as for estimation of influence of impulse and vibration loadings on pipelines. 


\section{REFERENCES}

[1] O. S. Limarchenko, G. Matarazzo, V. V. Yasinskiy, Rotatonal motion of structures with tanks partially filled by liquid, FADA Ltd, Kiev, 2003, 288 p.

[2] Yu. E. Vasilevskiy, O. S. Limarchenko, Nonlinear model of pipeline dynamics for high-speed liquid flow, Complex analysis and free boundary flows, Proceedings of Institute of Mathematics NAS of Ukraine 2 (4), Institute of Mathematics NAS of Ukraine, Kiev, 2006, P. 322-334.

[3] S. G. Mikhlin, Variational methods of mathematical physics [in Russian], Nauka, Moscow, 1970, $512 \mathrm{p}$.

[4] I. M. Babakov, Theory of oscillations, [in Russian], Nauka, Moscow, 1968, 560 h.

[5] N. G. Bondar, Nonlinear autonomous problems of mechanics of elastic systems [in Russian], Budivelnyk, Kiev, 1971, 265 p.

[6] V. I. Fedoseyev, On oscillations and stability of pipe, when liquid flows in it, Engineering collection 10 (1951) 251-257.

[7] Yu. A. Mitropolskiy, O. S. Limarchenko, G. Matarazzo, L. Toscano, Mathematical modeling of elastic systems with unilateral external interaction, Problems of control and informatics, 2001, No. 5, P. 48-70.

[8] O. Limarchenko, G. Matarazzo, A. Fedullo, Nonlinear mechanism of purposeful energy redistribution for suppression of oscillations of structures on moving foundation, Complex analysis and free boundary flows, Proceedings of Institute of Mathematics NAS of Ukraine, Vol. 1, No. 3, Institute of Mathematics NAS of Ukraine, Kiev, 2004, P. 136-143.

Received April 21, 2009

\section{ĐộNG LỰC HỌC PHI TUYẾN ĐƯờNG ỐNG CHỨA CHẤT LỎNG CHẢY VỚI VẬN TỐC LÂN CẬN VẬN TỐC TỚI HẠN}

Chúng ta đã phát triển mô hình phi tuyến của động lực học đường ống chứa chất lỏng chảy với vận tốc cao. Trên cơ sở của các phương pháp khác nhau chúng ta đã xây dựng được mô hình rời rạc phi tuyến và thuật toán số để giải bài toán động lực học và ổn định động học của đường ống. Chúng ta đã xét các ví dụ của ứng xử động học của hệ với các vận tốc dòng chảy khác nhau của chất lỏng, bao gồm trường hợp vận tốc giới hạn, khi có sự mất ổn định đường thẳng của hệ ống. 